\title{
On Black Hole Creation in Planckian Energy Scattering
}

\author{
I.Ya. Aref'eva ${ }^{*}$ K.S. Viswanathan `and I.V.Volovich ${ }^{\ddagger}$
}

\begin{abstract}
In a series of papers Amati, Ciafaloni and Veneziano and 't Hooft conjectured that black holes occur in the collision of two light particles at planckian energies. In this talk based on [10] we discuss a possible scenario for such a process by using the Chandrasekhar-Ferrari-Xanthopoulos duality between the Kerr black hole solution and colliding plane gravitational waves.
\end{abstract}

\section{Introduction}

An idea by M.A.Markov [1, 2], see also [3], that black holes can be considered as elementary particles becomes nowadays a common place in quantum gravity [4] and superstring theory [5, 6, 7]. If black holes are particles then one can ask about creation and annihilation of black holes in scattering processes of ordinary particles with very high energies. Amati, Ciafaloni and Veneziano [8] and 't Hooft [9] made a conjecture that black holes will occur in the collision of two light particles at Planck energies with small impact parameters.

In this talk based on [10] we discuss the following possible mechanism of black holes creation

\section{Particles $\rightarrow$ gravitational waves $\rightarrow$ Black Holes}

Ultra-relativistic particles generate plane gravitational waves then these plane gravitational waves collide and produce a singularity or a black hole.

We are going to discuss some issues concerning quantum mechanical description of black hole creation in the scattering processes of particles. Note that black holes cannot be incorporated into the theory if we consider quantum field theory in Minkowski space-time.

It is difficult to perform calculations for such a process in a realistic situation. We shall discuss here an idealized picture. Any gravitational wave far away from sources can be

*Steklov Mathematical Institute, Vavilov st.42, GSP-1, 117966, Moscow , Russia; E-mail : arefeva@arevol.mian.su

${ }^{\dagger}$ Department of Physics, Simon Fraser University, Burnaby, British Columbia, V5A 1S6, Canada, E-mail: kviswana@sfu.ca 
considered as a plane wave. We assume that plane waves already have been produced by ultrarelativistic particles and then consider analytically the process of black hole formation under interaction of these plane waves.

We discuss the semiclassical transition amplitude for the process of creation of black hole in the collision of two plane waves in the semiclassical approximation. For this purpose use a classical discribtion of the process

\section{gravitational waves $\rightarrow$ Black Holes}

Classical collision of plane gravitational waves has been the subject of numerous investigations, see for example [16, 17, 18, 19], and it has a remarkably rich structure. Here we are going to use the Chandrasekhar-Ferrari-Xanthopoulos duality between colliding plane gravitational waves and the Kerr black hole solution.

One has two dimensionless parameters in a scattering process at Planck energies [8, 9]. If $E$ is the energy in the center of mass frame, then one defines the Planckian energy regime to be $G E^{2} / \hbar \geq 1$, where $G$ is the Newton constant. This means that one can treat the process semiclassically. The other dimensionless parameter is $G E / b$, where $b$ is the impact parameter. If one takes $G E / b \ll 1$, one can use the eikonal approximation. The elastic scattering amplitude $A(s, t)$ in the eikonal approximation was found in 8, 9]. Fabbrichesi, Pettorino, Veneziano and Vilkovisky (FPVV) [15] gave the representation

$$
A(s, t) \sim s \int d^{2} b e^{i q b} e^{i I_{c l}(s, b)}
$$

Here $s$ and $t$ are the Mandelstam variables, $q^{2}=-t . I_{c l}(s, b)$ was taken to be the value of the boundary term for the gravitational action calculated on the sum of two Aichelburg-Sexl shock waves,

$$
I_{c l}(s, b)=G s \log b^{2} .
$$

This corresponds to a linearization of the problem and one cannot see in such an approximation black hole formation. It is tempting to suggest that one can use the FPVV approach [15] and the formula of type (1) even for small impact parameters to calculate the phase of the transition amplitude from a state containing two particles to a state containing a black hole. In such a case $I_{c l}(s, b)$ could be the value of the gravitational action for a solution of Einstein equation corresponding to an interior of the Kerr black hole created in the collision of plane gravitational waves. The parameters $s$ and $b$ in this case could be expressed in terms of the mass and angular momentum of the Kerr black hole.

\section{Black Hole Transition Amplitude}

We discuss here some issues concerning quantum mechanical description of black hole creation in the scattering processes of particles. Let us note first that black holes cannot be incorporated into the theory if we consider quantum field theory in Minkowski space-time. In fact it is obvious from Einstein equation

$$
R_{\mu \nu}-\frac{1}{2} g_{\mu \nu} R=8 \pi G T_{\mu \nu}
$$


in some approximation. Then, in the process of collision, one gets a strong gravitational field including perhaps black holes and/or singularities. Let us clarify the meaning of the transition amplitude from a state describing particles to a state containing black holes,

$$
<\text { Black holes } \mid \text { Particles in almost Minkowski space-time }>\text {. }
$$

By analogy with the definition of transition amplitude in quantum mechanics this transition amplitude can be characterized by values of the metric and others fields in two given moments of time (or by data on two given Cauchy surfaces, say $\Sigma^{\prime}$ and $\Sigma^{\prime \prime}$ ).

Our starting point is the quantum-mechanical Feynman transition amplitude between definite configurations of the three-metric $h_{i j}^{\prime}$ and field $\Phi^{\prime}$ on an initial spacelike surface $\Sigma^{\prime}$ and a configuration $h_{i j}^{\prime \prime}$ and $\Phi^{\prime \prime}$ on a final surface $\Sigma^{\prime \prime}$. This is

$$
<h^{\prime \prime}, \phi^{\prime \prime}, \Sigma^{\prime \prime} \mid h^{\prime}, \phi^{\prime}, \Sigma^{\prime}>=\int e^{\frac{i}{\hbar} S[g, \Phi]} \mathcal{D} \Phi \mathcal{D} g
$$

where the integral is over all four-geometries and field configurations which match given values on two spacelike surfaces, i.e.

$$
\begin{gathered}
\left.\Phi\right|_{\Sigma^{\prime}}=\phi^{\prime},\left.g\right|_{\Sigma^{\prime}}=h^{\prime} \\
\left.\Phi\right|_{\Sigma^{\prime \prime}}=\phi^{\prime \prime},\left.g\right|_{\Sigma^{\prime \prime}}=h^{\prime \prime}
\end{gathered}
$$

In this paper we shall consider the transition amplitude in the semiclassical approximation and we don't discuss here the introduction of ghosts and the definition of the measure $D g$.

We are interested in the process of black hole creation. Therefore we specify the initial configuration $h^{\prime}$ and $\phi^{\prime}$ on $\Sigma^{\prime}$ as configuration of gravitational and matter fields in Minkowski spacetime and we specify the final configuration $h^{\prime \prime}$ and $\phi^{\prime \prime}$ on $\Sigma^{\prime \prime}$ as describing a black hole. So, $\Sigma^{\prime}$ is a partial Cauchy surface with asymptotically simple past in a strongly asymptotically predictable space-time and $\Sigma^{\prime \prime}$ is a partial Cauchy surface containing black hole(s), i.e. $\Sigma^{\prime \prime}-J^{-}\left(\mathcal{T}^{+}\right)$is non empty. To explain these let us recall some necessary notions from the theory of black holes [21, 22].

Black holes are conventionally defined in asymptotically flat space-times by the existence of an event horizon $H$. The horizon $H$ is the boundary $\dot{J}^{-}\left(\mathcal{I}^{+}\right)$of the causal past $J^{-}\left(\mathcal{I}^{+}\right)$of future null infinity $\mathcal{I}^{+}$, i.e. it is the boundary of the set of events in space-time from which one can escape to infinity in the future direction.

The black hole region $B$ is

$$
B=M-J^{-}\left(\mathcal{I}^{+}\right)
$$

and the event horizon

$$
H=\dot{J}^{-}\left(\mathcal{T}^{+}\right)
$$

Consider a space that is asymptotically flat in the sense of being weakly asymptotically simple and empty, that is, near future and past null infinities it has a conformal structure like that of Minkowski space-time. One assumes that space-time is future asymptotically predictable, i.e. there is a surface $\mathcal{S}$ in spacetime that serves as a Cauchy surface for a region extending to future null infinity. This means that there are no "naked singularities" (a singularity that can be seen from infinity) to the future of the surface $\mathcal{S}$. This gives a formulation of Penrose's cosmic censorship conjecture. 
(i) $\tilde{M}-\mathcal{I}$ is conformal to $M$ with $\tilde{g}_{\mu \nu}=\Omega^{2} g_{\mu \nu}$,

(ii) $\Omega>0$ in $\tilde{M}-\mathcal{T}$ and $\Omega=0$ on $\mathcal{I}$ with $\nabla_{\mu} \Omega \neq 0$ on $\mathcal{I}$,

(iii) Every null geodesic on $\tilde{M}$ contains, if maximally extended, two end points on $\mathcal{I}$.

If $M$ satisfies the Einstein vacuum equations near $\mathcal{I}$ then $\mathcal{I}$ is null. $\mathcal{I}$ consists of two disjoint pieces $\mathcal{I}^{+}$(future null infinity) and $\mathcal{I}^{-}$(past null infinity) each topologically is $\approx R \times S^{2}$.

A space-time $M$ is weakly asymptotically simple if there exists an asymptotically simple $M_{0}$ with corresponding $\tilde{M}_{0}$ such that for some open subset $\mathrm{K}$ of $\tilde{M}_{0}$ including $\mathcal{I}$, the region $M_{0} \cup K$ is isometric to an open subset of $M$. This allows $M$ to have more infinities than just $\mathcal{I}$.

The domain of dependence $D^{+}(\Sigma)\left(D^{-}(\Sigma)\right)$ of a set $\Sigma$ is defined as the set of all points $p \in M$ such that every past (future) inextendible non-spacelike curve through $p$ intersects $\Sigma$. A space like hypersurface which no non-spacelike curve intersects more than once is called a partial Cauchy surface. Define $D(\Sigma)=D^{+}(\Sigma) \cup D^{-}(\Sigma)$. A partial Cauchy surface $\Sigma$ is said to be a global Cauchy surface if $D(\Sigma)=M$.

Let $\Sigma$ be a partial Cauchy surface in a weakly asymptotically simple and empty spacetime $(M, g)$. The space-time $(M, g)$ is (future) asymptotically predictable from $\Sigma$ if $\mathcal{I}^{+}$is contained in the closure of $D^{+}(\Sigma)$ in $\tilde{M}_{0}$. If, also, $J^{+}(\Sigma) \cap \bar{J}^{-}\left(\mathcal{I}^{+}, \bar{M}\right)$ is contained in $D^{+}(\Sigma)$ then the space-time $(M, g)$ is called strongly asymptotically predictable from $\Sigma$. In such a space there exist a family $\Sigma(\tau), 0<\tau<\infty$, of spacelike surfaces homeomorphic to $\Sigma$ which cover $D^{+}(\Sigma)-\Sigma$ and intersects $\mathcal{I}^{+}$. One could regard them as surfaces of constant time. A black hole on the surface $\Sigma(\tau)$ is a connected component of the set

$$
B(\tau)=\Sigma(\tau)-J^{-}\left(\mathcal{I}^{+}, \bar{M}\right) .
$$

One is interested primarily in black holes which form from an initially non-singular state. Such a state can be described by using the partial Cauchy surface $\Sigma$ which has an asymptotically simple past, i.e. the causal past $J^{-}(\Sigma)$ is isometric to the region $J^{-}(\mathcal{I})$ of some asymptotically simple and empty space-time with a Cauchy surface $\mathcal{I}$. Then $\Sigma$ has the topology $R^{3}$.

In the case considered one has a space-time $\left(M, g_{\mu \nu}\right)$ which is weakly asymptotically simple and empty and strongly asymptotically predictable .

$\Sigma^{\prime}$ is a partial Cauchy surface with asymptotically simple past, $\Sigma^{\prime} \sim R^{3}$.

$\Sigma^{\prime \prime}=\Sigma\left(\tau^{\prime \prime}\right)$ contains a black hole, i.e. $\Sigma^{\prime \prime}-J^{-}\left(\mathcal{I}^{+}, \bar{M}\right)$ is nonempty.

In particular, if one has the condition $\Sigma^{\prime} \cap \bar{J}^{-}\left(\mathcal{I}\right.$ ) is homeomorphic to $R^{3}$ (an open set with compact closure) then $\Sigma^{\prime \prime}$ also satisfies this condition.

Strictly speaking one cannot apply the standard definition of black holes to the case of plane gravitational waves and we need a generalization. One defines a black hole in terms of the event horizon, $\dot{J}^{-}\left(\mathcal{I}^{+}\right)$. However this definition depends on the whole future behaviour of the metric. There is a different sort of horizon which depends only on the properties of spacetime on the surface $\Sigma(\tau)$ 21]. Any point in the black hole region bounded by $r=2 m$ in the Kruskal diagram represents a trapped surface (which is a two-dimensional sphere in spacetime) in that both the outgoing and ingoing families of null geodesics emitted from this point converge and hence no light ray comes out of this region. A generalization of the definition of black holes in terms of trapped horizon has been considered in 26, 29. A generalization 
We discussed the transition amplitude (propagator) between definite configurations of fields, $<h^{\prime \prime}, \phi^{\prime \prime}, \Sigma^{\prime \prime} \mid h^{\prime}, \phi^{\prime}, \Sigma^{\prime}>$. The transition amplitude from a state described by the wave function $\Psi^{\text {in }}\left[h^{\prime}, \phi^{\prime}\right]$ to a state $\Psi^{\text {out }}\left[h^{\prime \prime}, \phi^{\prime \prime}\right]$ reads

$$
\begin{gathered}
<\Psi^{\text {out }} \mid \Psi^{\text {in }}>= \\
\int \bar{\Psi}^{\text {out }}\left[h^{\prime \prime}, \phi^{\prime \prime}\right]<h^{\prime \prime}, \phi^{\prime \prime}, \Sigma^{\prime \prime} \mid h^{\prime}, \phi^{\prime}, \Sigma^{\prime}>\Psi^{\text {in }}\left[h^{\prime}, \phi^{\prime}\right] \mathcal{D} h^{\prime} \mathcal{D} \phi^{\prime} \mathcal{D} h^{\prime \prime} \mathcal{D} \phi^{\prime \prime} .
\end{gathered}
$$

One can take for example the state $\Psi^{i n}=\Psi_{p_{1} p_{2}}^{i n}\left[h^{\prime}, \phi^{\prime}\right]$ as a Gaussian distribution describing a state of particles with momenta $p_{1}$ and $p_{2}$ and take $\Psi^{\text {out }}$ as a wave function describing a state of black hole. Recently Barvinski, Frolov and Zelnikov have suggested an expression for the wave function of the ground state of a black hole [20].

\section{Boundary term in Gravitational Action and Semi- classical Expansion}

In this section we discuss an approximation scheme for calculating the transition amplitude following the FPVV approach [15]. The gravitational action with the boundary term has the form [23]

$$
S[g]=-\frac{1}{16 \pi G} \int_{V} R \sqrt{-g} d^{4} x-\frac{1}{8 \pi G} \int_{\partial V} K \sqrt{h} d^{3} x
$$

Here $V$ is a domain in space-time with the space-like boundary $\partial V, h$ is the first fundamental form and $K$ is the trace of the second fundamental form of $\partial V$.

The case of null surfaces was considered in [24]. We shall write a representation of the boundary term suitable for quantum consideration. The action is

$$
S[g]=-\frac{1}{16 \pi G}\left(\int_{V} d^{4} x \sqrt{-g} R(g)+\int_{V} d^{4} x \sqrt{-g} \nabla_{\mu} f^{\mu}(g)\right),
$$

where

$$
f^{\mu}(g)=g^{\alpha \beta} g^{\mu \nu} \partial_{\nu} g_{\alpha \beta}-g^{\mu \alpha} g^{\beta \nu} \partial_{\nu} g_{\alpha \beta} .
$$

Supposing that the boundary is described by equation

$$
\sigma(x)=0
$$

one gets the action in the form

$$
S[g]=-\frac{1}{16 \pi G}\left(\int_{V} d^{4} x \sqrt{-g} R(g)+\int_{V} d^{4} x \sqrt{-g_{c l}} \delta(\sigma(x)) f^{\mu} \nabla_{\mu} \sigma\right) .
$$

The linearization of the action (3.2) leads to the action in the FPVV form [15].

Let us show that the presence of the boundary term in the action (3.2) is necessary for the selfconsistency of the semiclassical expansion. To perform semiclassical expansion, one expands the metric $g$ around a classical solution $g_{c l}$ of the Einstein equation so that $g=g_{c l}+\delta g$, 
where $g_{c l}$ matches the given Cauchy data on surfaces $\Sigma^{\prime}$ and $\Sigma^{\prime \prime}$, i.e.

$$
\left.g_{c l}\right|_{\Sigma^{\prime}}=h^{\prime},\left.\quad g_{c l}\right|_{\Sigma^{\prime \prime}}=h^{\prime \prime}
$$

In this case $\left.\delta g\right|_{\Sigma^{\prime}}=\left.\delta g\right|_{\Sigma^{\prime \prime}}=0$, but we cannot guarantee that $\left.\nabla \delta g\right|_{\Sigma^{\prime}}=\left.\nabla \delta g\right|_{\Sigma^{\prime \prime}}=0$. To ensure that terms linear in $\nabla \delta g$ drop out from expression (3.6) (otherwise we cannot perform semiclassical expansion) one has to integrate by parts. One has

$$
\begin{gathered}
S[g+\delta g]=-\frac{1}{16 \pi G}\left(\int d^{4} x \sqrt{-g} R(g)+\int d^{4} x \sqrt{-g} \nabla_{\mu} f^{\mu}(g)\right)+ \\
\left.+\int d^{4} x \sqrt{-g}\left(g^{\mu \nu} \nabla^{2} \delta g_{\mu \nu}-\nabla^{\mu} \nabla^{\nu} \delta g_{\mu \nu}\right)+\nabla_{\mu}\left(g^{\alpha \beta} \partial^{\mu} \delta g_{\alpha \beta}-g^{\mu \alpha} \partial^{\beta} \delta g_{\alpha \beta}\right)\right) \\
+ \text { second order terms. }
\end{gathered}
$$

In eq.(3.8) we have dropped the subscript $c l$ from $g_{c l}$. The linear terms coming from the Hilbert-Einstein action can be put in the form

$$
g^{\mu \nu} g^{\alpha \beta} \nabla_{\alpha} \nabla_{\beta} \delta g_{\mu \nu}-\nabla^{\mu} \nabla^{\nu} \delta g_{\mu \nu}=\nabla_{\mu}\left(g^{\alpha \beta} \nabla^{\mu} \delta g_{\alpha \beta}-g^{\mu \alpha} \nabla^{\beta} \delta g_{\alpha \beta}\right)
$$

Notice that on the RHS of the last relation covariant derivatives can be replaced by partial derivatives because on the boundary $\delta g=0$. Therefore one finds that terms linear in $\delta \partial g$ coming from Hilbert-Einstein action cancel similar terms coming from full divergence and the action (2.2) admits the expansion (3.6).

Taking into account that the value of the Hilbert-Einstein action on the classical solution is equal to zero, one finds that the full action for a solution of Einstein equation is reduced to the second term in (3.8) which can be reduced to a boundary term.

The transition amplitude (2.2) in semiclassical approximation is

$$
<h^{\prime \prime}, \Sigma^{\prime \prime} \mid h^{\prime}, \Sigma^{\prime}>=\mathcal{Z} \exp \frac{i}{\hbar} S_{c l}
$$

where

$$
S\left[g_{c l}\right]=-\frac{1}{16 \pi G} \int d^{4} x \sqrt{-g_{c l}} \delta(\sigma(x)) f^{\mu} \nabla_{\mu} \sigma,
$$

$f$ is given by equation (3.3) and

$$
\mathcal{Z}=\left(\frac{\pi}{\operatorname{det} S^{\prime \prime}\left(g_{c l}\right)}\right)^{1 / 2}
$$

We assume here that there is only one solution of classical equation of motion with given boundary conditions.

\section{Colliding Plane Gravitational Waves}

There exists a well known class of plane-fronted gravitational waves with the metric

$$
d s^{2}=2 d u d v+h(u, X, Y) d u^{2}-d X^{2}-d Y^{2}
$$

where $u$ and $v$ are null coordinates. In particular the gravitational field of a particle moving with the speed of light is given by the Axelburg-Sexl solution. The metric has the form 


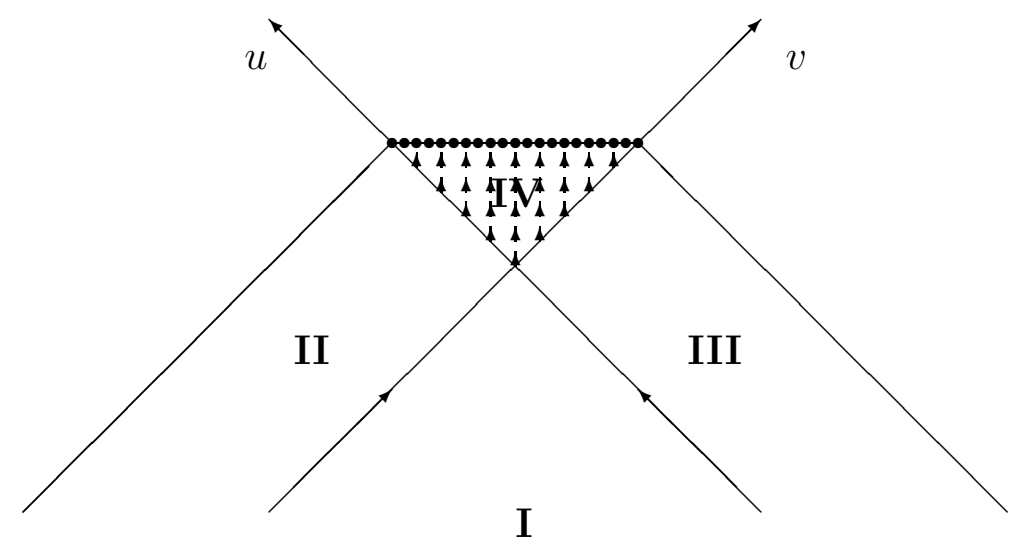

Figure 1: $(u, v)$ plane wave coordinates

and describes a shock wave. It is difficult to find a solution which describes two sources. An approximate solution of equation (2.1) for two particles as the sum of solutions each of which describes one particle was considered by FPVV[15]. This approximation describes well the scattering amplitude for large impact parameter, but does not describe non-linear interaction of shock waves which is dominant in the region of small impact parameter. To analyze non-linear effects we will, instead of dealing with shock waves, take a simple solution of Einstein equation, namely we will take plane gravitational waves. In some respects one can consider plane wave as an approximation to more complicated gravitational waves, in particular shock waves. This solution in some sense can be interpreted as an approximation for a solution of Einstein equation in the presence of two moving particles. Collision of two ultrarelativistic black holes was considered by D'Eath [25]. If our particles are gravitons then there are no sources corresponding to matter fields in Einstein equations. Note also that plane gravitational waves are produced by domain walls, see [12].

A particular class of plane waves is defined to be plane-fronted waves in which the field components are the same at every point of the wave surface. This condition requires that $h(u, X, Y)$ is a function with a quadratic dependence on $X$ and $Y$. One can then remove the dependence of $h$ on $X$ and $Y$ altogether by a coordinate change. Solutions of Einstein equations describing collision of plane gravitational waves were first obtained by Szekeres and Khan and Penrose [16]. Chandrasekhar, Ferrari and Xanthopoulos [17, 18] have developed a powerful method for obtaining such solutions by using a remarkable analogy ("duality") with stationary axisymmetric case which can be reduced to the investigation of the Ernst equation. For a review see the Griffiths book [19].

We will use the coordinates $(u, v, x, y)$. We assume that throughout space-time there exists a pair of commuting space-like Killing vectors $\xi_{1}=\partial_{x}, \xi_{2}=\partial_{y}$. The Szekeres line element has the form

$$
d s^{2}=2 e^{-N} d u d v-e^{-U}\left(e^{V} \cosh W d x^{2}-2 \sinh W d x d y+e^{-V} \cosh W d y^{2}\right),
$$

Here $N, U, V$ and $W$ are functions of $u$ and $v$ only.

We illustrate in Fig. 1 the two-dimensional geometry of plane waves. Space-time is divided into four regions. The region $\mathbf{I}$ is the flat background before the arrival of the plane waves. 
plane waves which interact in region IV. Colliding plane gravitational waves can produce singularities or Cauchy horizons in the interaction region [26, 17, 27, 28, 29]. The solution is undetermined across a Cauchy horizon [17, 30, 29, 31] into the future. We shall discuss two simplest extensions.

In particular, one can get an interior of the Schwarzschild solution in the interaction region IV.There are two types of colliding plane waves solutions corresponding to the Schwarzschild metric. The first one creates the interior of the black hole with the usual curvature singularity. In this case incoming plane waves have curvature singularities already before collision. In the context of the Planck energy scattering it seems more natural that we don't have curvature singularities already for free plane gravitational waves. Therefore we will be discussing mainly another type of solutions one gets in the interaction region, namely, the interior of the Schwarzschild white hole. The maximal analytic extension of this solution across its Killing-Cauchy horizon leads to creation of a covering space of the Schwarzschild black hole out of collision between two plane gravitational waves. An alternative interpretation of this solution is the creation of the usual Schwarzschild black hole out of the collision between two plane gravitational waves propagating in a cylindrical universe. There exists also a timereversed extension [29] including the covering space of the Schwarzschild exterior and part of black hole, and giving two receding plane waves with flat space between. We will interpret this as scattering of plane waves on the virtual black hole.

Vacuum Einstein equations for the metric (4.3) give a system of differential equations for functions $U, V, N$ and $W$. One of these equations can be integrated to give (see [10] for details)

$$
e^{-U}=f(u)+g(v)
$$

where $f(u)$ and $g(v)$ are arbitrary functions. We fix a gauge by using $f=f(u)$ and $g=g(v)$ as new coordinates instead of $u$ and $v$ and then we change variables from $f$ and $g$ to $\mu$ and $\eta$ such that

$$
f+g=\left(1-\mu^{2}\right)^{1 / 2}\left(1-\eta^{2}\right)^{1 / 2}, \quad f-g=-\mu \eta .
$$

By introducing the complex valued function

$$
Z=\chi+i \lambda
$$

where

$$
\chi=e^{-V} / \cosh W, \quad \lambda=e^{-V} \tanh W .
$$

one can reduce the system of differential equations for functions $U, V, N$ and $W$ to the Ernst equation on $Z$. In particular, this procedure gives the following solution of the vacuum Einstein equations

$$
d s^{2}=X\left(\frac{d \eta^{2}}{1-\eta^{2}}-\frac{d \mu^{2}}{1-\mu^{2}}\right)-\left(1-\mu^{2}\right)^{1 / 2}\left(1-\eta^{2}\right)^{1 / 2}\left[\chi d y^{2}+\frac{1}{\chi}(d x-\lambda d y)^{2}\right],
$$

where

$$
\begin{gathered}
\chi=\left(1-\mu^{2}\right)^{1 / 2}\left(1-\eta^{2}\right)^{1 / 2} \frac{X}{Y} \\
\lambda=\frac{2 q}{p}\left[\frac{1}{1+p}-\frac{\left(1-\eta^{2}\right)(1-p \mu)}{1-p^{2} \mu^{2}-q^{2} \eta^{2}}\right]
\end{gathered}
$$


$p$ and $q$ satysfy

$$
E=p \mu+i q \eta
$$

Now let us take

$$
p=-\frac{\left(m^{2}-a^{2}\right)^{1 / 2}}{m}, \quad q=\frac{a}{m}, m \geq a
$$

and introduce the new coordinates $(t, r, \theta, \phi)$ instead of $(\mu, \eta, x, y)$ by putting

$$
\begin{gathered}
\mu=\frac{r-m}{\left(m^{2}-a^{2}\right)^{1 / 2}}, \quad \eta=\cos \theta \\
t=-\sqrt{2} m\left(x-\frac{2 q}{p(1+p)}\right), \quad \phi=\frac{\sqrt{2} m}{\left(m^{2}-a^{2}\right)^{1 / 2}} y
\end{gathered}
$$

Then the metric (4.8) will take the form of the Kerr solution

$2 m^{2} d s^{2}=\left(1-\frac{2 m r}{\rho^{2}}\right) d t^{2}-\frac{4 a m r}{\rho^{2}} \sin ^{2} \theta d t d \phi-\left(r^{2}+a^{2}-\frac{2 a^{2} m r}{\rho^{2}}\right) \sin ^{2} \theta d \phi^{2}-\rho^{2}\left(\frac{1}{\Delta} d r^{2}+d \theta^{2}\right)$,

where

$$
\rho^{2}=r^{2}+a^{2} \cos ^{2} \theta, \quad \Delta=r^{2}-2 m r+a^{2}
$$

The coordinates must satisfy the inequality $|\eta|<\mu \leq 1$. This implies that $-\left(m^{2}-a^{2}\right) \sin ^{2} \theta<$ $\Delta \leq 0$, which means the region of the Kerr spacetime that is inside the ergosphere.

To describe the colliding plane gravitational waves producing the interior of the ergosphere in the Kerr spacetime it is convenient to rewrite the metric (4.8) in terms of the $\left(u^{\prime}, v^{\prime}\right)$ coordinates related with $(\eta, \mu)$ by the following relations

$$
\eta=\sin \left(u^{\prime}-v^{\prime}\right), \quad \mu=\sin \left(u^{\prime}+v^{\prime}\right),
$$

These $\left(u^{\prime}, v^{\prime}\right)$ are related to $(u, v)$ in (4.3) by $u=\sin u^{\prime}, v=\sin v^{\prime}$. For simplicity of notations we will omit' in (4.18). We have

$$
d s^{2}=4 X(u, v) d u d v-\Omega(u, v)\left[\chi(u, v) d y^{2}+\frac{1}{\chi(u, v)}(d x-\lambda(u, v) d y)^{2}\right]
$$

where

$$
\begin{gathered}
X(u, v)=(1-p \sin (u+v))^{2}+q^{2} \sin ^{2}(u-v), \quad \Omega(u, v)=\cos (u+v) \cos (u-v), \\
\lambda(u, v)=\frac{2 q}{1+p} \frac{1-\sin (u+v)}{Y(u, v)}\left((p+1) \sin ^{2}(u-v)+p \sin (u+v)-1\right) \\
Y(u, v)=1-p^{2} \sin ^{2}(u+v)-q^{2} \sin ^{2}(u-v) .
\end{gathered}
$$

$\operatorname{In}(4.19)$

$$
0<u<\pi / 2, \quad 0<v<\pi / 2, \quad v+u<\pi / 2
$$

To extend the metric (4.19) outside of region (4.23) one uses the Penrose-Khan trick and substitutes in (4.19) 


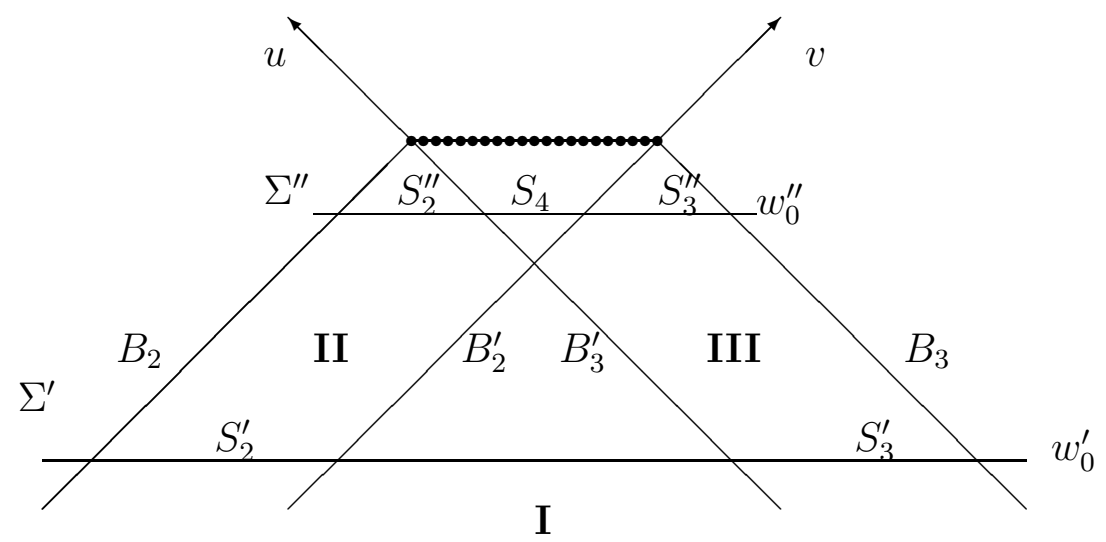

Figure 2: Initial and final Cauchy surfaces

$$
\theta(x)= \begin{cases}1, & x>0 \\ 0, & x<0\end{cases}
$$

Fig.1 illustrates this metric. The region $\mathbf{I}(u<0, v<0)$ is Minkowskian. Regions II and III contain the approaching plane waves with the following metrics

$$
\begin{aligned}
& \left(d s^{I I}\right)^{2}=4 X(u) d u d v-\Omega(u)\left[\chi(u) d y^{2}+\frac{1}{\chi(u)}(d x-\lambda(u) d y)^{2}\right], \\
& \left(d s^{I I}\right)^{2}=4 X(v) d u d v-\Omega(v)\left[\chi(v) d y^{2}+\frac{1}{\chi(v)}(d x-\lambda(v) d y)^{2}\right],
\end{aligned}
$$

where

$$
\begin{gathered}
X(u)=(1-2 p \sin u)+\sin ^{2} u, \quad \Omega(u)=\cos ^{2} u, \\
\lambda(u)=2 q\left(\sin u-\frac{1}{1+p}\right)
\end{gathered}
$$

In 4.26$)$

$$
u<\pi / 2, \quad v<0
$$

and in 4.27$)$

$$
u<0, \quad v<\pi / 2,
$$

The region $\mathbf{I V}$ is the interaction region with metric (4.19).

\section{Semiclassical Transition Amplitude}

In this section we study the transition amplitude

$$
<2 p w, W H \mid 2 p w>
$$

from a state $\mid 2 p w>$ of two plane gravitational waves to a state $\mid 2 p w, W H>$ containing white-hole and two plane gravitational waves. Analogous calculations may be performed for 
from two plane gravitational waves back to two plane gravitational waves and for the amplitude

$$
<B H \mid 2 p w>
$$

for a process 2 plane waves $\rightarrow$ black holes. We can consider these two amplitudes as amplitudes of two independent channels.

To find these transition amplitudes in the semiclassical approximation according to (3.10), we have to evaluate the boundary term for the classical solution interpolating between two plane gravitational initial state and an appropriate final state.

Let us start considering the transition (5.1). The corresponding classical solution is shown in Fig.2. In this case the initial Cauchy surface $\Sigma^{\prime}$ crosses regions I,II and III and the final surface crosses regions II,IV and III. For the surfaces $\Sigma^{\prime}$ and $\Sigma^{\prime \prime}$ shown in Fig.2 equation (3.4) reads

$$
\begin{gathered}
\Sigma^{\prime}: \sigma=u+v-w_{0}^{\prime}=0, w_{0}^{\prime} \leq 0 \\
\Sigma^{\prime \prime}: \sigma=u+v-w_{0}^{\prime \prime}=0,0<w_{0}^{\prime \prime} \leq \pi / 2
\end{gathered}
$$

In this case the other parts of the boundary defining the boundary term are given by equations

$$
\begin{aligned}
& B_{2}: u-\pi / 2=0, w_{0}^{\prime}-\pi / 2 \leq v \leq w_{0}^{\prime \prime}-\pi / 2 ; \quad B_{2}^{\prime}: u=0, \quad w_{0}^{\prime}-\pi / 2 \leq v \leq 0 \\
& B_{3}: v-\pi / 2=0, w_{0}^{\prime}-\pi / 2 \leq u \leq w_{0}^{\prime \prime}-\pi / 2 ; \quad B_{3}^{\prime}: v=0, \quad w_{0}^{\prime}-\pi / 2 \leq u \leq 0
\end{aligned}
$$

To calculate the phase factor let us give expressions for $f^{\mu}$ on the boundary. In Minkowski space we have $f^{\mu}=0$. In all regions $f^{x}=f^{y}=0$, moreover

$$
\left.f^{u}\right|_{I I}=0,\left.\quad f^{v}\right|_{I I I}=0
$$

So the value of the action (3.11) for the two plane waves solution (4.26), 4.27) is reduced to the sum of two terms each of which represents a contribution from the Cauchy surface $\Sigma^{\prime}$ and $\Sigma^{\prime \prime}$, respectively,

$$
S\left(g_{c l}^{(2 p w)}\right)=S^{\Sigma^{\prime}}+S^{\Sigma^{\prime \prime}}
$$

where

$$
S^{\Sigma^{\prime}}=S_{2}^{\prime}+S_{3}^{\prime}, \quad S^{\Sigma^{\prime \prime}}=S_{2}^{\prime \prime}+S_{3}^{\prime \prime}+S_{4}^{\prime \prime}
$$

see Fig.2. So

$$
<2 p w \mid 2 p w, W H>=\mathcal{Z} \exp \left\{\frac{i}{\hbar}\left(S_{2}^{\prime}+S_{3}^{\prime}-S_{2}^{\prime \prime}-S_{3}^{\prime \prime}-S_{4}^{\prime \prime}\right)\right\}
$$

here $\mathcal{Z}$ is the one-loop contribution.

Taking into account the special form of the metric one can write $f^{w}$ in regions II, III and $\mathbf{I V}$ respectively in the following form

$$
\begin{gathered}
\left.\sqrt{-g} f^{w}\right|_{I I}=\Omega(u) \partial_{u} \ln \left[X(u) \Omega^{2}(u)\right] \\
\left.\sqrt{-g} f^{w}\right|_{I I I}=\Omega(v) \partial_{v} \ln \left[X(v) \Omega^{2}(v)\right] \\
\left.\sqrt{-g} f^{w}\right|_{I V}=\Omega(w, z) \partial_{w} \ln \left[X(w, z) \Omega^{2}(w, z)\right]
\end{gathered}
$$


The representation (5.12) was obtained in the following way. Metric (4.19) in $(w, z, x, y)$ coordinates admits the representation

$$
g_{\mu \nu}=\left(\begin{array}{cc}
g_{\alpha \beta} & 0 \\
0 & g_{i j}
\end{array}\right) .
$$

with the diagonal matrix $g_{\alpha \beta}$,

$$
g_{\alpha \beta}=\left(\begin{array}{cc}
g_{w w} & 0 \\
0 & g_{z z}
\end{array}\right) ., \quad g_{w w}=-g_{z z}=X
$$

and $g_{i j}$ of the form

$$
g_{i j}=\Omega \tilde{g_{i j}}
$$

where

$$
\tilde{g_{i j}}=\left(\begin{array}{cc}
\frac{1}{\chi} & -\frac{\lambda}{\chi} \\
\frac{\lambda}{\chi} & \chi+\frac{\lambda^{2}}{\chi}
\end{array}\right) .
$$

being an element of the group $S L(2)$. From equation (3.3) it follows

$$
f^{w}=g^{w w}\left(g^{z z} \partial_{w} g_{w w}+g^{i j} \partial_{w} g_{i j}\right)
$$

Taking into account that the trace $\operatorname{tr} \tilde{g}^{-1} \partial \tilde{g}=0$ for the group $S L(2)$, we get

$$
f^{w}=g^{w w}\left(g^{z z} \partial_{w} g_{w w}+2 \Omega^{-1} \partial_{w} \Omega\right)
$$

from which follows the representation (5.14).

We have to compute the value of the boundary term in the action on the plane wave solution. Because the plane wave metric does not depend on $x$ and $y$ coordinates we have to introduce a cut-off $L$ in these directions. Simple calculations give

$$
\begin{gathered}
S_{2}^{\prime}=-\frac{1}{16 \pi G} \int_{-L / 2}^{L / 2} d x \int_{-L / 2}^{L / 2} d y \int_{\pi / 2}^{0} d u \int_{w_{0}^{\prime}-\pi / 2}^{w_{0}^{\prime}} d v \delta\left(u+v-w_{0}^{\prime}\right) \Omega(u) \partial_{u} \ln \left[X(u) \Omega^{2}(u)\right] \\
=-\frac{1}{16 \pi G} L^{2} \int_{\pi / 2}^{0} d u \cos ^{2} u \partial_{u}\left[\ln \left((1-p \sin u)^{2}+q^{2} \sin ^{2} u\right) \cos ^{4} u\right] \\
=-\frac{2 L^{2}}{16 \pi G}\left(\int_{1}^{0} d u \frac{\left(1-u^{2}\right)(u-p)}{1-2 u p+u^{2}}-4 \int_{1}^{0} u d u\right) \\
=-\frac{L^{2}}{16 \pi G}\left(-3-2 p+2 q^{2} \ln 2(1-p)+4 p q \arctan \frac{q}{1-p}\right)
\end{gathered}
$$

and

$$
S_{2}^{\prime \prime}=-S_{2}^{\prime}+\bar{S}_{2}^{\prime \prime}
$$

where

$$
\begin{gathered}
\bar{S}_{2}^{\prime \prime}=\frac{L^{2}}{16 \pi G} \int_{w_{0}^{\prime \prime}}^{0} d u \cos ^{2} u \partial_{u}\left[\ln \left((1-p \sin u)^{2}+q^{2} \sin ^{2} u\right) \cos ^{4} u\right] \\
=\frac{L^{2}}{16 \pi G}\left[-3 \sin ^{2} w_{0}^{\prime \prime}-2 p \sin w_{0}^{\prime \prime}+2 q^{2} \ln \left(\sin ^{2} w_{0}^{\prime \prime}-2 p \sin w_{0}^{\prime \prime}+1\right)+4 p q \arctan \frac{q \sin w_{0}^{\prime \prime}}{1-p \sin w_{0}^{\prime \prime}}\right]
\end{gathered}
$$


some constant of dimension of square of the length, in particular, we can take $c=m^{2}$. If we take metric $\tilde{g}_{\mu \nu}$ in our computations of the transition amplitude then $L^{2}$ in (5.21) and (5.23) disappears.

The part of the boundary which cross the white hole region gives the following contribution

$$
\begin{aligned}
& S_{4}^{\prime \prime}=-\frac{1}{16 \pi G} \int_{-L / 2}^{L / 2} d x \int_{-L / 2}^{L / 2} d y \int_{-w_{0}^{\prime}}^{w_{0}^{\prime}} d z \Omega(w, z) \partial_{w} \ln \left[X(w, z) \Omega^{2}(w, z)\right] \\
& =-\frac{L^{2}}{16 \pi G} \int_{w_{0}^{\prime \prime}}^{-w_{0}^{\prime \prime}} d z\left[\frac{-2 p\left(1-p \sin w_{0}^{\prime \prime}\right) \cos ^{2} w_{0}^{\prime \prime} \cos z}{\left(1-p \sin w_{0}^{\prime \prime}\right)^{2}+q^{2} \sin ^{2} z}-2 \sin w_{0}^{\prime \prime} \cos z\right]
\end{aligned}
$$

Now let us consider the special case when $w_{0}^{\prime \prime}=\pi / 2$. In this case we do not have plane waves in the final state but only a white hole. Then only the second term in the RHS of (5.24) survives and we get

$$
S^{\Sigma^{\prime \prime}}=S_{4}^{\prime \prime}=\frac{L^{2}}{4 \pi G}
$$

Therefore the amplitude describing the transition from two plane waves to white hole in the semiclassical approximation is given by

$$
\begin{gathered}
<W H \mid 2 p w>=\mathcal{Z} \exp \left\{\frac{i}{\hbar}\left(S_{2}^{\prime}+S_{3}^{\prime}-S_{4}^{\prime \prime}\right)\right\}= \\
\mathcal{Z} \exp \left\{-\frac{i L^{2}}{8 \pi G \hbar}\left[-1-2 p+2 q^{2} \ln 2(1-p)+4 p q \arctan \frac{q}{1-p}\right]\right\}
\end{gathered}
$$

To get the semiclassical answer for the transition amplitude $<2 p w \mid 2 p w>$ we have to consider the classical solution which describes the metric extended as shown in Fig.4. We see from equations (5.21) that $S_{2}^{\prime}$ does not depend on $w_{0}^{\prime}$ and therefore for the transition amplitude describing the elastic scattering of two plane waves the phase factors is zero, and

$$
<2 p w \mid 2 p w>=\mathcal{Z}
$$

In this section we have computed only the phases of transition amplitudes. However they contain a nontrivial information about our processes because we different channels available and there would be a quantum mechanical interference between them.

\section{CONCLUSION}

We have considered a possible mechanism for black hole creation in the collision of two light particles at planckian energies. Many questions deserve further study. In particular there is an important question of how to relate the momenta of colliding particles with characteristics of colliding plane waves. It is known that a plane wave does not lead to polarization of vacuum [34. It was shown that a plane wave is an exact string background [35, 36, 37, 38] and there is a duality with extreme black hole [39]. One can conjecture that a dilaton gravity analogue of the CFX duality between colliding plane waves and non-extremal black holes discussed in this paper could lead to a corresponding string duality. 


\section{ACKNOWLEDGMENT}

This work has been supported in part by an operating grant from the Natural Sciences and Engineering Research Council of Canada. I.A. and I.V. thank the Department of Physics for kind hospitality during their stay at Simon Fraser University. I.A. and I.V. are supported in part also by International Science Foundation under the grant M1L300. I.V. is grateful to D.Amati, A.Barvinsky, W.Israel, V.Frolov, D.Page, G.Veneziano and A.Zelnikov for stimulating and critical discussions.

\section{References}

[1] M.A.Markov, Progr.Theor.Phys.Suppl.1(1965)247;

[2] M.A.Marov, Reflecting on Universe, preprint INR 0892/95, (1995).

[3] V.P.Frolov and I.D.Novikov, Physics of Black Holes, Nordrecht, 1987.

[4] S.W.Hawking, Virtual Black holes, Preprint DAMTP/R-95/50(1995).

[5] A.Sen, Extremal Black Holes and Elementary String States, hep-th/9504147.

[6] E.Witten, String Theory Dynamics in Various Dimensions, hep-th/9503124.

[7] C.Hall and P.Townsend, Unity of Superstring Dualities, QMW-94-30, R/94/33.

[8] D.Amati, M.Ciafaloni and G.Veneziano, Phys.Lett. B197(1987)81;

Phys.Lett. B216 (1989) 41; Int.J.Mod.Phys. A3 (1988) 1615; Nucl.Phys. B347 (1990) 550; Nucl.Phys. B403 (1993) 703.

[9] G.'t Hooft, Phys.Lett. B198 (1987); Nucl.Phys. B304 (1988) 867;

[10] I.Ya. Aref'eva, K.S. Viswanathan and I.V.Volovich, Nucl.Phys.B 452346

[11] I.Muzinich and M.Soldate, Phys.Rev.D37 (1988) 353

[12] C.O.Lousto and N.Sanchez, Phys.Lett.232 (1989) 462; Nucl. Phys.B355(1991)231

[13] E.Verlinde and H.Verlinde, Nucl.Phys.B371 (1992) 246

[14] D.Kabat and M.Ortiz, Nucl.Phys.B388 (1992) 570

[15] M.Fabbrichesi, R.Pettorino, G.Veneziano and G.Vilkovisky, Nucl.Phys. B419(1994)174

[16] P.Szekeres, Nature,228(1970)1183; J.Math.Phys.13 (1972) 286

K.A.Khan and R.Penrose, Nature,229(1971)185

[17] S.Chandrasekhar and B.Xanthopoulos, Proc. R.soc.A 408 (1986) 175; A410 (1987) 311

[18] V.Ferrari and J.Ibanez, Gen.Rel.Grav.19 (1987) 1053

[19] J.B.Griffiths, Colliding Plane Waves in General Relativity, Clarendon Press,1991 
[21] S.W.Hawking and G.R.F.Ellis, The large scale structure of space-time. Cambridge University Press, 1973

[22] R.M.Wald, General Relativity. The University of Chicago Press, Chicago,1984

[23] J.W.York,Jr., Phys.Rev.Lett.28(1772)1082

T.Regge and C.Teitelboim, Ann.Phys.(N.Y.)88(1974)286

G.W.Gibbons and S.W.Hawking, Phys.RevD15(1977)2752

Y.Choquet-Bruhat, A.Fisher and J.Marsden, in Proc.Enrico Fermi Summer School of the Italian Physical Society, Varenna, ed. J.Ehlers (1978)

[24] C.Barrabas and W.Israel, Phys.Rev.D43(1991)1129

[25] P.D.D'Eath, Phys.Rev.D18(1978)990

[26] R.A.Matzner and F.J.Tipler, Phys.Rev.D29(1984)1575

[27] U.Yurtsever, Phys.Rev.D38(1988)1706

[28] D.A.Konkowski and T.M.Helliwell, Class.Quantum Grav. 6(1989)1847

[29] S.A.Hayward, Class.Quantum Grav.6(1989)1021

[30] U.Yurtsever, Phys.Rev.D37(1988)2790

[31] C.Hoenselaers and F.J.Ernst, J.Math.Phys.31(1990)144

[32] U.Yurtsever, Phys.Rev.D40(1989)360

[33] M.Dorca and E.Verdaguer, Nucl.Phys.B403(1993)770

[34] G.Gibbons, Comm.Math.Phys.42(1986)435

[35] D.Amati and C.Klimcik, Phys.Lett. B219(1989)443

[36] C.Nappi and E.Witten, Phys.Rev.Lett.71(1993)3751

[37] G.T.Horowitz and A.A.Tseytlin, Preprint, Imperial/TP-93-94/51

[38] A.A.Tseytlin, hep-th/9407099

[39] E.Bergshoeff, R.Kallosh and T.Ortin, hep-th/9406009 